\title{
Tübinger Modell der Anamnesegruppen
}

\author{
Zwischen Kommunikationstraining und Selbsterfahrung
}

Autoren

Institut

\author{
K. E. Keifenheim, M. Teufel, E. R. Petzold
}

Abteilung für Psychosomatische Medizin und Psychotherapie, Medizinische Klinik, Universitätsklinikum Tübingen

\section{Schlüsselwörter \\ - Anamnesegruppen \\ Arzt-Patient-Kommunikation \\ - patientenorientierte Medizin \\ - Medizinstudierende \\ - Peer-gestütztes Lernen}

Key words

peer-groups on historytaking

- doctor-patient communication

- patient-oriented medicine

- medical students

peer-assisted learning

\section{Bibliografie}

DOI http://dx.doi.org/

10.1055/s-0034-1375644

Balint 2014; 15: 56-60

(c) Georg Thieme Verlag KG

Stuttgart · New York

ISSN 1439-5142

Korrespondenzadresse

Dr. med. Katharina Eva

Keifenheim

Abteilung Innere Medizin VI

Psychosomatische Medizin und

Psychotherapie

Medizinische Universitätsklinik

Osianderstraße 5

72076 Tübingen

katharina.keifenheim@med.

uni-tuebingen.de

\section{Zusammenfassung \\ $\nabla$}

Im Medizinstudium liegt die Betonung heute auf klinischem (Praxis-)Bezug und frühzeitigem Patientenkontakt bereits in vorklinischen Semestern. Es wird vermehrt in Kleingruppen gearbeitet und klinisch-praktische Fertigkeiten der ArztPatient-Kommunikation werden an den meisten Fakultäten zunehmend vermittelt. Die Aufgabe des Arztes, „Kommunikator“ zu sein, wurde in den zentralen CanMEDS-Rollen ${ }^{1}$ festgeschrieben und hat so Eingang in den Nationalen Kompetenzbasierten Lernzielkatalog (NKLM) gefunden. Welche Bedeutung hat diese Entwicklung für die Anamnesegruppen, die in Deutschland seit nunmehr rund 40 Jahren in studentischer Selbstverwaltung existieren? Der Artikel beschreibt das Modell der Tübinger Anamnesegruppen heute und skizziert Herausforderungen und Chancen vor dem Hintergrund aktueller Entwicklungen in der medizinischen Lehre. Exemplarisch wird der Fallbericht einer Medizinstudierenden (5. Fachsemester) aus dem Sommersemester 2013 vorgestellt.

\section{Einleitung}

$\nabla$

Als sich die Anamnesegruppen in den 70er und 80er Jahren entwickelten und ausgehend von Ulm, Heidelberg und Marburg in ganz Deutschland verbreiteten, entsprang dies dem Wunsch der Studierenden nach einem frühzeitigen Kontakt mit Patienten und nach einer ganzheitlichen Herangehensweise im Sinne des bio-psycho-sozialen Modells.

In Tübingen wurde Stephan Zipfel im Jahre 2004 Nachfolger von Heinz Henseler auf dem Lehrstuhl für Psychosomatische Medizin und Psycho-

${ }^{1}$ Die 7 CanMEDS-Rollen, unter ihnen der Arzt als „Kommunikator“, beschreiben zentrale Rollen im Berufsbild des Arztes. Sie sind inzwischen international anerkannt und auch Herzstück des deutschen NKLM.

\section{Abstract \\ $\nabla$}

In today's medical education, clinical practice and early contact to patients in undergraduate education are emphasized. Faculties increasingly make use of working in small groups. Imparting of clinical skills and doctor-patient communication is more and more implemented. The physician's role as a "communicator" is defined in the CanMEDS Roles and has found its way into the National Competence - Based Learning Objectives for Undergraduate Medical Education in Germany (NKLM). What does this development mean for "peer groups for taking medical histories" (anamnesis groups), which exist in Germany in student self-administration for over 40 years now? This article describes the Tübingen model for peer groups on taking medical histories today and outlines challenges and chances against the background of current developments in medical education. As an example, a report by a medical student of the $5^{\text {th }}$ semester from summer term of 2013 is presented. therapie der Eberhard-Karls-Universität. Ebenfalls 2004 nahm Markus Schrauth (1975-2008), ein junger Assistenzarzt dieser Klinik, Kontakt mit dem Autor (ERP) auf. Als Student in Marburg hatte Schrauth die dortigen Anamnesegruppen kennen und schätzen gelernt, wusste aber auch, wie hilfreich eine Supervision für die Fragen der Tutoren sein kann. Dies entsprach den Erfahrungen Petzolds, seitdem er sich Ende der siebziger Jahre in Heidelberg auf Anamnesegruppen eingelassen hatte [2].

Zum Sommersemester 2005 wurde mit den Anamnesegruppen in Tübingen begonnen, nicht ohne Betonung der studentischen Selbstorganisation, die natürlich auch Arbeit bedeutete. Anamnesegruppen wie auch Selbstorganisation waren hier etwas Ungewöhnliches. In der Fakultät 
schien man den Verzicht auf klare Strukturen zu fürchten und dass die Studierenden den Patienten schaden könnten - weit gefehlt, wer das denkt, sitzt einem gewaltigen Irrtum auf.

Selbstorganisiertes Lernen hat im Gegensatz zu dem von außen gesteuerten Lernen eine ganz andere Kreativität und Kraft. Längst ist bekannt, dass der Wahlspruch der Aufklärung, „Sapere aude!" $=$ Habe Mut $z u$ deinem eigenen Verstand durch „Sentire aude! " $=$ Habe Mut zu deinen eigenen Gefühlen zu ergänzen ist. Die Orientierung an den eigenen Gefühlen und der „inneren Stimme“ erweitert die Fähigkeit des Lernens. Dafür ist Raum zu schaffen. Wir waren und sind bis heute überzeugt: Der Emotionalität Frei- und Spielraum zu geben, ist ein wesentlicher Beitrag auf dem Weg, eine gute Ärztin oder ein guter Arzt zu werden.

Die Arzt-Patient-Kommunikation wurde über Jahrzehnte hauptsächlich hier, in Anamnesegruppen und fernab des regulären Curriculums, vermittelt und geübt. Veranstaltungen zur Anamneseerhebung, wie sie an den meisten medizinischen Fakultäten heute verpflichtend angeboten werden, vermitteln neben einer Anamnesegrundstruktur, die zu einer vollständigen Erhebung aller relevanten Daten beitragen soll, vor allem Fragetechniken. Es handelt sich um sehr heterogene Unterrichtsformate, die häufig in Seminaren oder Kleingruppen stattfinden. Häufig wird die Anamneseerhebung in Rollenspielen oder mit Schauspielpatienten geübt, manchmal kommen auch reale Patienten zum Einsatz. Das sogenannte „peer-assisted learning“ (PAL) mit studentischen Tutoren scheint sich vor allem im englischsprachigen Raum zu etablieren.

Was können Anamnesegruppen ihren heutigen Studierenden bieten, was möglicherweise nach wie vor überwiegend abseits der Pflichtveranstaltungen zu finden ist? Und was bedeutet es für Teilnehmer und Mitwirkende, wenn Anamnesegruppen über ihre Anerkennung als Wahlfach Anbindung an das Curriculum erfahren? Dieser Artikel beschreibt das Tübinger Modell der Anamnesegruppen und schildert die Herausforderungen und Chancen, vor denen alle Beteiligten stehen, wenn ein solches Format als benotetes Wahlpflichtfach angeboten wird und so Anschluss an das Curriculum findet.

\section{Tübinger Modell der Anamnesegruppen}

Eine Anamnesegruppe besteht aus sieben bis acht Teilnehmern², von denen einer die Anamnese erhebt, und zwei studentischen Tutoren. Eine erste Tübinger Besonderheit: Die Teilnehmer sind zu etwa gleichen Teilen Studierende der Medizin und der Psychologie, dasselbe gilt für das Tutorentandem, das sich aus je einem Medizin- und einem Psychologiestudierenden zusammensetzt. Die Gruppe trifft sich über ein Semester einmal wöchentlich abends in einem Seminarraum in einer der Tübinger Universitätskliniken. Ziel zu Beginn ist ein gegenseitiger Austausch zur eigenen derzeitigen Situation, was in Form eines Blitzlichts erfolgt. Gedanken oder Nachträge zur letzten Stunde sowie in der Zwischenzeit aufgekommene Fragen werden besprochen. Im nächsten Schritt wird der am Vortag von einem der Teilnehmer ausgewählte Patient in seinem Zimmer abgeholt. Im Seminarraum findet die Anamnese vor der Gruppe statt. In Ausnahmefällen, wenn Patienten nicht mobil sind, findet die Anam-

\footnotetext{
${ }^{2}$ Im Folgenden wird jeweils die männliche Form gewählt, gemeint sind selbstverständlich immer beide Geschlechter gleichermaßen.
}

nese auch im Patientenzimmer statt. Zu Beginn wird auf den Rahmen hingewiesen - dass nun einer der Teilnehmer ein Anamnesegespräch führen wird, dass alle Teilnehmer der Schweigepflicht unterliegen und dass der Patient auch die Freiheit hat, Fragen nicht zu beantworten. Die Themenkomplexe im Leitfaden für das Anamnesegespräch orientieren sich an der Anamnese nach Adler und Hemmeler [3] und versuchen, „Zugang zu den biologischen, psychischen und sozialen Aspekten“ zu ermöglichen. Nachdem der Anamneseführende das Gespräch beendet hat, wird - das Einverständnis des Patienten vorausgesetzt - die Runde für die Gruppe geöffnet, die nun ergänzende Fragen stellen kann. Danach begleitet ein Teilnehmer den Patienten zurück in sein Zimmer. Es findet anschließend ein strukturiertes Feedback der Gruppe an den Anamneseführenden statt, welches durch die Tutoren moderiert wird. Ein Fokus liegt hierbei auf wertschätzender und konstruktiver Formulierung des Feedbacks. Inhaltlich beziehen sich die Rückmeldungen auf die Vollständigkeit und Struktur der Anamnese, auf verbale Kommunikation (z.B. Frageformen, Begrüßung und Verabschiedung, Gestaltung von Übergängen), aber auch auf nonverbale und emotionale Signale des Patienten und des Anamneseführenden, auf Empathie, Wertschätzung und Passung im Gespräch. Auch die Tutoren geben dem Anamneseführenden ein Feedback. Dann wird in der Gruppe über die Anamnese und den Patienten gesprochen. Dabei geht es mehr um das Verhalten des Anamneseführenden und weniger um die Patientengeschichte. Ziel der Diskussion ist eine umfassende Diagnose im Sinne Michael Balints [4-7], die auch biografische Daten und Beziehungsaspekte mit einbezieht. Zum Abschluss der Gruppe findet erneut ein Blitzlicht statt, in dem die Mitglieder ihre Eindrücke, Gedanken oder ihre Stimmung mitteilen können. Es gibt in Tübingen seit dem Sommersemester 2012 die Möglichkeit, sich die Teilnahme an der Anamnesegruppe als Wahlfach anerkennen zu lassen. In diesem Fall schreibt der Anamneseführende im Anschluss einen Reflexionsbericht, zu dem er von seinen Tutoren und den Supervisoren (KEK und ERP) eine individuelle Rückmeldung bekommt. Die Tutoren sind Studierende der Medizin oder Psychologie, die mindestens ein Semester lang selbst an einer Anamnesegruppe teilgenommen haben. Sie besuchen zu Beginn ihrer Tutorentätigkeit eine didaktische Schulung ( $\bullet$ Abb. 1), angeboten durch das Kompetenzzentrum für Medizindidaktik der Fakultät $[7,8]$ sowie eine fachliche Schulung ( $\bullet$ Abb. 2), angeboten durch die Autorin (KEK). Während des Semesters finden fortlaufend zweiwöchentliche Supervisionen mit den Autoren (ERP und KEK) statt, in denen die Tutoren aus ihren Gruppen berichten. Fragen und Anliegen der Tutoren werden besprochen. Einmal im Semester findet mit den Tutoren außerdem ein Workshop zur Einführung in die Balintarbeit statt (ERP).

\section{Die Didaktikschulung für Tutoren besteht aus 5 Modulen:}

1. Effektiv präsentieren

2. Präzise erklären

3. Spontane Lehreinheiten gestalten

4. Vermitteln klinischer Fertigkeiten mit Hilfe der Peyton-Methode

5. Umgang mit schwierigen (Gruppen-) Situationen

Abb. 1 Didaktikschulungen für Tutoren. 


\section{Inhalte der fachlichen Schulung für Tutoren sind:}

1. Gesprächsführung

- Grundstruktur einer Anamnese

- Nonverbale Kommunikation

- Aktives Zuhören

2. Interaktion mit Teilnehmern

- Gemeinsam Gesprächssituationen reflektieren

- Feedback

3. Selbstreflektion

- Respekt vor Individualität und subjektiver Wahrnehmung anderer

- Erkennen eigener Verhaltensweisen

Abb. 2 Fachliche Schulung für Tutoren.

\section{Erwartungen an die Anamnesegruppe und an die Supervision \\ $\nabla$}

Teilnehmer und Tutoren werden jeweils zu Beginn und Ende eines Semesters mithilfe von Fragebögen nach einer Selbsteinschätzung ihrer Anamnesefertigkeiten sowie nach Einstellungen und Erwartungen an die Anamnesegruppe und an die Supervision befragt. ${ }^{3}$ Es zeigt sich die Tendenz, dass beide, Tutoren wie Teilnehmer, sich zu Beginn des Semesters durch die Teilnahme an Anamnesegruppen vor allem Sicherheit in der Struktur und Vollständigkeit der Anamnese erhoffen und die Vermittlung grundlegender Gesprächs- und Fragetechniken erwarten. Daneben steht bei den Studierenden, die sich in den ersten Semestern ihres Studiums in der Regel mit dem Erwerb theoretischen Wissens beschäftigen und nicht mit Patienten arbeiten, der Wunsch nach Patientenkontakt im Vordergrund. Insbesondere nach dem Besuch einer Anamnesegruppe über mindestens ein Semester zeigt sich vermehrt der Wunsch nach Hilfestellung im Umgang mit schwierigen Gesprächssituationen. Als solche werden die Erhebung einer vegetativen Anamnese inklusive der Sexualanamnese und das Ansprechen heikler Themen, z.B. Sterben und Tod oder Suizidalität, genannt, der Umgang mit starken Emotionen im Gespräch (ärgerliche, unzufriedene oder weinende Patienten) sowie der Umgang mit „interaktionell herausfordernden“, bspw. sehr mitteilsamen oder sehr schweigsamen Patienten. Zu diesem Zeitpunkt wünschen sich Teilnehmer vermehrt auch Rückmeldungen zu ihrem Auftreten und nonverbalen Verhalten im Kontakt mit Patienten, welche sie in den Anamnesegruppen als hilfreich erlebt haben.

\section{Anerkennung der Anamnesegruppen als Wahlfach $\nabla$}

Die Belastung der Medizinstudierenden durch zeitintensive Curricula, Veranstaltungen auch am Abend, Anwesenheitspflicht in Seminaren und Kursen sowie durch die Notwendigkeit, in allen Fächern benotete Leistungsnachweise zu erbringen, ist seit dem Inkrafttreten der neuen Approbationsordnung [9] 2003 deutlich

\footnotetext{
${ }^{3}$ Projekt pGROWTH (peer-assisted, GROup-oriented Way of Teaching History-taking). Keifenheim, K. E. et al., Publikation in Vorbereitung.
}

angestiegen. Korrespondierend hat sich seit 2004 die Teilnehmerstruktur der Tübinger Anamnesegruppen mit leichten Schwankungen kontinuierlich verändert. Im Lauf der Jahre meldeten sich deutlich mehr Psychologie- als Medizinstudierende zu den Anamnesegruppen an. Auch bei den Tutoren waren die Medizinstudierenden schließlich in der Unterzahl. An der medizinischen Fakultät Tübingen wurden etwa ab 2009 vermehrt studentische Tutoren insbesondere zur Vermittlung klinischpraktischer Fertigkeiten eingesetzt und es wurden hierfür auch Anreize geschaffen [10]. Es wurde deutlich, dass eine Gleichstellung der Anamnesegruppentutoren mit ihren studentischen Tutorenkollegen, die etwa im Präparierkurs oder im „skills lab“ tätig waren, notwendig war, um das Bestehen der Anamnesegruppen für Medizinstudierende zu sichern und eine Teilnahme attraktiver zu gestalten.

Seit dem Sommersemester 2012 sind Tutoren der Tübinger Anamnesegruppen in das Programm „Docendo discimus“ der Medizinischen Fakultät eingebunden. Sie sind somit als studentische Hilfskräfte angestellt und erhalten für ihre Arbeit eine Vergütung. Qualitätssichernde Maßnahmen wie Schulungen und Supervisionen, die in Tübingen seit jeher zum Standard der Anamnesegruppen gehörten, sind nun verbindlich. Für die Teilnehmer wird der Besuch einer Anamnesegruppe über ein Semester als Wahlfach mit 20 Stunden anerkannt und auf der Grundlage des Reflexionsberichtes auch benotet. Für Studierende des klinischen Studienabschnitts ist der Schein außerdem mit 20 Stunden auf das Wahlfach Psychosomatische Medizin und Psychotherapie anrechenbar. Daneben ist es jedoch weiterhin möglich, unabhängig von einer Wahlfachanerkennung teilzunehmen, auch über mehrere Semester hinweg. Für die Psychologiestudierenden ist ersteres ohnehin nach wie vor die Regel, da ein Besuch der Anamnesegruppe für sie derzeit nicht anrechenbar ist.

Die Anbindung an das Curriculum über die Anerkennung als Wahlpflichtveranstaltung ist ein Novum in der nun über 40-jährigen Tradition der Anamnesegruppen, die aus studentischer Initiative entstanden und über Jahrzehnte in studentischer Selbstverwaltung gewachsen sind. Bisher galten Anamnesegruppen als „guided self-help“, angeleitete Selbsthilfe, und boten Studierenden die Chance, einen Teil ihrer Ausbildung selbst zu bestimmen und Verantwortung für die Bildung ihrer (Arzt-)Persönlichkeit, ihrer beruflichen Identität, zu übernehmen. Die Aufgabe der Fakultät war es, eine Supervision bereitzustellen und Zugang zu geeigneten Räumen sowie zu Patienten der verschiedenen Abteilungen zu ermöglichen. Die Anerkennung der Anamnesegruppen als Wahlfach hat zahlreiche Implikationen. Für die Teilnehmer bedeutet sie, dass ihr Engagement abgebildet und ihr Einsatz dem ihrer Kommilitonen in anderen Wahlfächern gleichgestellt wird. Für die Tutoren bedeutet es ebenso eine Gleichstellung mit studentischen Tutorenkollegen und eine Vergütung ihrer Arbeit, außerdem einen Anspruch auf Schulung und Supervision. Daneben aber erhalten die Anamnesegruppen stärker als in der Vergangenheit eine Anbindung an das Fach Psychosomatische Medizin und Psychotherapie und eine inhaltliche wie institutionelle Kontinuität. Die bisher ausschließlich externe Supervision wird in Tübingen nun durch einen erfahrenen externen Supervisor (ERP) und eine Ärztin in Weiterbildung im Fach Psychosomatische Medizin und Psychotherapie (KEK) gemeinsam gestaltet. Durch die Wahlfachanerkennung besteht für die Teilnehmer der Anreiz, im klinischen Abschnitt des Studiums weitere Wahlveranstaltungen auf dem Gebiet der Psychosomatischen Medizin zu besuchen. Die Teilnehmerzahlen haben 
sich seit der Wahlfachanerkennung auf einem stabilen Niveau von 20 bis 30 Teilnehmern pro Semester gehalten und Medizinstudierende sind als Tutoren und als Teilnehmer wieder zu gleichen Teilen wie Psychologiestudierende in den Gruppen vertreten. Durch den engeren Kontakt mit dem Fach Psychosomatik interessieren sich Teilnehmer und Tutoren vermehrt für Dissertationen, Famulaturen und Praktika. Die Anamnesegruppen bedeuten so auch Nachwuchsförderung im Bereich Psychosomatische Medizin.

Ein Format, das Studierende aus Vorklinik und Klinik, aus verschiedenen Semestern und unterschiedlichen Studiengängen gemeinsam unterrichten möchte, bietet zahlreiche Chancen, hat aber auch so manche organisatorische Hürde zu nehmen. Ein Wahlpflichtfach anzubieten, das von studentischen Tutoren geleitet wird, bedeutet eine Herausforderung, etwa in der Frage nach der Benotung der Teilnehmer. Eine Anbindung der Anamnesegruppen an das Curriculum kann langfristig eine vermehrte Verschulung zur Folge haben. Diese Verschulung könnte zu einer Reduktion der Verantwortung führen, die Teilnehmer und Tutoren für ihr Format tragen. Anamnesegruppen bedeuten nicht nur Selbstorganisation, sondern auch Selbstwirksamkeit und Selbststeuerung. Eine ausschließlich studentische Selbstorganisation und -verwaltung aber ist bei einem Wahlpflichtfach nicht mehr möglich. Dennoch erachten die Autoren es als wichtig, den Tutoren so viel Verantwortung und Entscheidungsfreiheit wie möglich zu übertragen. Letzteres auch aus einem anderen Grund: Anamnesegruppen boten ihren Teilnehmern immer einen geschützten Raum zur Entwicklung und Reflexion, der nicht von der Fakultät dominiert wurde und in dem es möglich war, eine eigene professionelle und persönliche Identität $\mathrm{zu}$ formen [11] - und diese Chance soll erhalten bleiben. Auch hier ist es wohl ratsam, den Rat Michael Balints zu beherzigen: „My only advice is don't be too ambitious and don't push them too hard. It is better to let them develop at their own pace." (Michael Balint in einem Brief an Boris Luban-Plozza, zitiert nach [12]).

\section{„Beyond content analysis and non-verbal behavior": Was bieten Anamnesegruppen heute? \\ $\nabla$}

Dass Anamnesegruppen nach 40 Jahren ihres Bestehens nun als Wahlfach anerkannt sind, fällt zeitlich zusammen mit einer Entwicklung, die Kommunikation sehr viel stärker als bisher im Curriculum verankert. Beispiele für diese Entwicklung sind die Novelle der Ärztlichen Approbationsordnung, die fordert, Kommunikation im Medizinstudium zu vermitteln und zu prüfen, ${ }^{4}$ die Arbeit am Nationalen Kompetenzbasierten Lernzielkatalog Medizin, der ärztlicher Kommunikation auch in den CanMEDRollen einen hohen Stellenwert zuschreibt $[13,14]$, sowie die Bestrebungen, ein Nationales Mustercurriculum Kommunikation zu erarbeiten [15]. Was können Anamnesegruppen ihren

\footnotetext{
${ }^{4}$ „In Anbetracht der großen Bedeutung sozialer und kommunikativer Fähigkeiten der Ärztin oder des Arztes für das Arzt-Patienten-Verhältnis und damit für eine erfolgreiche Krankenbehandlung („Sprechende Medizin“), sollten diese Aspekte verpflichtend Eingang in die ärztliche Ausbildung erhalten. Die Vermittlung ärztlicher Gesprächskompetenz soll daher als allgemeines Ziel der ärztlichen Ausbildung festgeschrieben werden. Darüber hinaus soll der Nachweis ärztlicher Gesprächskompetenz auch übergreifender Gegenstand der mündlich-praktischen Prüfung im Zweiten Abschnitt der Ärztlichen Prüfung (ab 1. Januar 2014: Dritter Abschnitt der Ärztlichen Prüfung) sein.“ Erste Verordnung zur Änderung der Approbationsordnung für Ärzte, 238/12. http://www.bundesrat.de/cln_320/nn_2291536/SharedDocs/Beratungsvorgaenge/2011/0801-900/0862-11.html
}

Mitgliedern ermöglichen, was dennoch so im Curriculum nicht vermittelt wird?

In einem bio-psycho-sozialen Modell gehen wir davon aus, dass es etwas gibt, das über die inhaltliche Vollständigkeit und professionelle Fragetechnik der Anamneseerhebung hinausgeht, und das nicht gelehrt werden kann, indem man es in immer kleinere Schritte zerlegt und diese auf einer Checkliste abhakt [16]. Eine Anamnese wird in der patientenorientierten Medizin verstanden als ein gemeinsames Erinnern, als eine persönliche Begegnung zwischen zwei Menschen, die von beiden ein Sich-Einlassen fordert [12]. Balint schreibt: „Einem Menschen zu begegnen, der Verständnis dafür hat, was man eigentlich möchte, und der sich offen gibt, ist so selten im Leben. Genau das ist es aber, was wir für unsere Patienten tun können, und dies ist ein gewaltiger Dienst. “ (Michael Balint in einem Brief an Boris Luban-Plozza 1965, aus dem Englischen übersetzt, zitiert nach [17]). Diesen Dienst aber können bereits Studierende ihren Patienten erweisen.

Die Arbeit in Anamnesegruppen bedeutet für ihre Mitglieder eine kontinuierliche Arbeit in einer allmählich vertrauter werdenden Kleingruppe. Es steht ausreichend Zeit zur Verfügung, um das zu entwickeln, was im Medizinstudium immer noch zu kurz kommt: eine ärztliche Haltung $[11,12,18]$ und patientenorientierte Einstellung. Als zentrale ärztliche Haltungen gelten unter anderem die personale Begegnung mit dem Patienten („helping alliance“), das Sich-Einlassen auf seine subjektive Krankheits- und Heilungstheorie, aber auch das In-VerbindungSein mit sich selbst und die Beziehungsgestaltung zu Kollegen und Angehörigen anderer Berufe [19]. Die geschützte Atmosphäre unter Peers ermöglicht eine ehrliche und konstruktive Rückmeldung zum eigenen Verhalten, ein Ausprobieren und ein Hineinwachsen in die eigene künftige Rolle.

Anamnesegruppen ermöglichen es, sich als Studierender als Person mit den eigenen Gefühlen angenommen zu fühlen - und nicht lediglich als „lebender Wissensspeicher“ oder „Lernmaschine“[18]. Sie fördern eine erweiterte Sichtweise der eigenen Person mit den individuellen Stärken und Schwächen. Eine professionelle Identität ist mit dem Tag der Approbation nicht einfach da, man muss in sie hineinwachsen wie in neue Schuhe, die anfänglich noch etwas zu groß sind. Patientenkontakte zu gestalten, muss gelernt und geübt werden, nicht anders als eine Herzkatheteruntersuchung oder eine Operation. Arzt-PatientKontakte, die als gelungen erlebt werden, sind sehr unterschiedlich - wichtig ist, dass sie authentisch sind [20]. Authentizität aber kann in Anamnesegruppen schrittweise erworben werden, denn das Feedback der Kommilitonen bildet ab, wenn Sprache und nonverbale Signale nicht zusammenpassen oder der Anamneseführende so wirkt, als fühle er sich mit dem Gesagten unwohl in seiner Haut. Anamnesegruppen fördern Emotionalität, Ausdrucksvermögen, Feingefühl und Empathie [17]. Vielleicht werden sie von Teilnehmern und Tutoren deshalb so oft als Ausgleich zum Studium beschrieben - als etwas, wo man „seinen Kopf frei bekommen kann“. Anamnesegruppen lehren, auf die eigene Wahrnehmung zu vertrauen, um das Lehrbuchwissen zu ergänzen. Sie lehren, auf die eigene Intuition zu hören, auf das eigene Gefühl für Angemessenheit und Atmosphäre, das in Sekundenbruchteilen darüber entscheidet, in welche Richtung sich eine Kommunikation weiter entwickelt - und ob sie Erfolg hat [16]. Nicht zuletzt zeigt das eigene Fühlen an, wo emotionale Konflikte beim Patienten liegen könnten.

Daneben vermitteln Anamnesegruppen auch die Übernahme von Verantwortung - der Anamneseführende trägt die Verantwortung für sein Gespräch mit dem Patienten, der Tutor die Ver- 
antwortung für das Gespräch in der Gruppe, die Teilnehmer der Gruppe die Verantwortung für sich und für den Umgang mit ihren Patienten. Etwas, das notwendig ist angesichts der hohen Verantwortung, die Ärzte und auch klinische Psychologen später haben werden, und die im Studium doch so selten adressiert wird. Nicht zuletzt tragen die Supervisoren Verantwortung für die Studierenden, für die Möglichkeiten des Anders-Seins, um die Besonderheiten dieses Formats zu wahren.

Markus Schrauth, einer der Initiatoren von Anamnesegruppen in Tübingen, verfasste bereits als Medizinstudent Gedichte. Während seiner Krankheit und in seiner letzten Lebensphase stellte er selbst den Band „Talgrund der Wünsche“ [21] zusammen. Mit dem folgenden Gedicht aus diesem Band möchten wir an ihn erinnern und zugleich den heutigen Studierenden Mut machen zur Suche nach einer eigenen - auch ärztlichen - Haltung in unserer Zeit.

warum eigentlich weiterstehen in der wüste den himmel anbrüllen und den dünen die welt erklären?

vielleicht weil da

noch andere rufer

die füße tief im treibsand

nach haltung suchen und

vielleicht weil wir alle

leiden an der zeit und

weil sie ohne uns

besser nicht wird.

Markus Schrauth

\section{Zur Person}

$\nabla$

K. E. Keifenheim ist Ärztin in Weiterbildung an der Medizinischen Universitätsklinik, Abteilung Psychosomatische Medizin und Psychotherapie, in Tübingen. Seit 2012 ist sie Supervisorin für Tutoren der Tübinger Anamnesegruppen und seit 2013 Lehrbeauftragte der Abteilung.

M. Teufel ist leitender Oberarzt an der Medizinischen Universitätsklinik, Abteilung Psychosomatische Medizin und Psychotherapie, in Tübingen. Engagiert in der Nachwuchsförderung und in der Entwicklung neuer Lehrformate z. B. Vorlesung zu „Allergie meets Psyche“ und „Rolle der Kommunikation in der PatientArzt-Beziehung“.

E. R. Petzold lebt nach seiner Emeritierung als Professor für Psychosomatische Medizin und Psychotherapie an den Universitäten Heidelberg, Homburg/Saar und Aachen in der Nähe von Tübingen. Supervision für Tutoren der Tübinger Anamnesegruppen seit 2005.
Ihre Meinung zu diesem Artikel können Sie gerne der Redaktion mitteilen (geschaeftsstelle@balintgesellschaft.de).

\section{Literatur}

1 Kröger F, Luban-Plozza B. Studenten-Balintgruppen - Eine Erweiterung der medizinischen Ausbildung. Patientenbezogene Medizin 1982; H. 4

2 Adler $R$, Hemmeler W. Praxis und Theorie der Anamnese. Der Zugang zu den biologischen, psychischen und sozialen Aspekten der Kranken. Stuttgart: 1992

3 Balint M. The doctor, his patient, and the illness. Lancet 1955; 268: 683-688

4 Balint M, Ball DH, Hare ML. Training medical students in patientcentered medicine. Compr Psychiatry 1969; 10: 249-258

5 Luban-Plozza B. A new training method -20 years of student Balint groups. Schweizerische Rundschau für Medizin Praxis=Revue suisse de medecine Praxis 1989; 78: 1192-1196

6 Luban-Plozza B. Empowerment techniques: from doctor-centered (Balint approach) to patient-centred discussion groups. Patient Educ Couns 1995; 26: 257-263

7 Heni M, Lammerding-Koppel M, Celebi $N$ et al. Focused didactic training for skills lab student tutors - which techniques are considered helpful? GMS Zeitschrift für medizinische Ausbildung 2012; 29: Doc41

8 Krautter M, Weyrich P, Schultz JH et al. Effects of Peyton's four-step approach on objective performance measures in technical skills training: a controlled trial. Teaching and learning in medicine 2011; 23: 244-250

9 Gesundheit Bf. Bundesgesetzblatt Jahrgang 2002 Teil I Nr. 44: Approbationsordnung für Ärzte vom 27. Juni 2002. In; 2002

10 Shiozawa T, Baur F. Docendo discimus - das neue Tutorensystem in Tübingen. Newsletter Studium und Lehre der Medizinischen Fakultät Tübingen 2009; 2/09

11 Schuffel $W$. Can medical students acquire patient centered attitudes at medical schools? Psychother Psychosom 1983; 40: 22-32

12 Petzold A, Petzold ER, Schüffel W. Anamnesegruppen - bewusst erlebte Sozialisation zum Arzt. In: Studt HH, Petzold ER, Hrsg. Psychotherapeutische Medizin Psychoanalyse - Psychosomatik - Psychotherapie Ein Leitfaden für Klinik und Praxis. Berlin, New York: de Gruyter; 1999; 434-439

13 Hibbeler B. Medizinstudium: Grundstein für den „guten Arzt“. Deutsches Ärzteblatt 2012; 109: A-844/B-731/C-727

14 Rourke J, Frank JR. Implementing the CanMEDSTM physician roles in rural specialist education: the Multi-Speciality Community Training Network. Rural and remote health 2005; 5: 406

15 Juenger J, Schultz JH, Köllner $V$ et al. Entwicklung eines nationalen, longitudinalen Mustercurriculums Kommunikation - Status und Ausblick. Jahrestagung der Gesellschaft für Medizinische Ausbildung (GMA): 2013; Graz

16 Langewitz $W$. Beyond content analysis and non-verbal behaviour what about atmosphere? A phenomenological approach. Patient Educ Couns 2007; 67: 319-323

17 Luban-Plozza B. Physician - where to? Schweizerische Rundschau für Medizin Praxis = Revue suisse de medecine Praxis 1993; 82: 677-685

18 Schuffel W, Egle U, Schneider A. Students speak with patients. Anamnesis groups as a form of education. MMW, Munchener medizinische Wochenschrift 1983; 125: 845-848

19 Rosin U. Das Medizinstudium. In: Studt HH, Petzold ER, Hrsg. Psychotherapeutische Medizin Psychoanalyse - Psychosomatik - Psychotherapie Ein Leitfaden für Klinik und Praxis. Berlin, New York: de Gruyter; 1999; 431-434

20 Luban-Plozza B. Encounter as therapy. Praxis 1995; 84: 853-858

21 Schrauth M. Talgrund der Wünsche. In Kassel: AQUINarte; 2007 